\title{
Haemodynamic parameters during cardiopulmonary resuscitation in estimating the degree of ischaemic contracture
}

Se-hyeok Park, Yong Deok Lim, Yong Hun Jung, Kyung Woon Jeung

Department of Emergency Medicine, Chonnam National University Hospital, Gwangju, Republic of Korea

Myocardial ischemic contracture, which refers to a progressive LV wall thickening following ischemia, occurs frequently in patients with prolonged cardiac arrest. Myocardial ischemic contracture inevitably results in reduction in the LV chamber volume. Thus, the volume of blood ejected by chest compression decreases as myocardial ischemic contracture progresses, ultimately precluding successful resuscitation (Fig. 1) In experimental settings, echocardiographic assessment with transesophageal or transmediastinal approaches has been used to observe the development of ischemic contracture during CPR. However, the echocardiographic assessments using these approaches are usually impractical or unavailable in clinical settings.

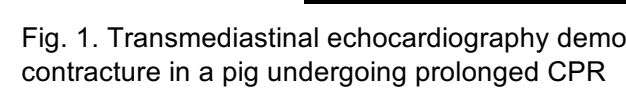

In the present study, we investigated, in a swine model of out-of-hospital cardiac arrest, whether hemodynamic parameters, including arterial pressure and ETCO2, can serve as predictive factors for the severity of ischemic contracture during CPR of consistent quality, and therefore can be utilized as a tool to estimate the severity of ischemic contracture during ACLS conducted according to resuscitation guidelines.

\section{Methods}

We retrospectively analyzed data derived from a previous study in Yorkshire/Landrace cross pigs weighing $25.2 \pm 2.9 \mathrm{~kg}$ (Fig. 2).

The IACUC of our university approved the study (CNU IACUC-H-2017-18).

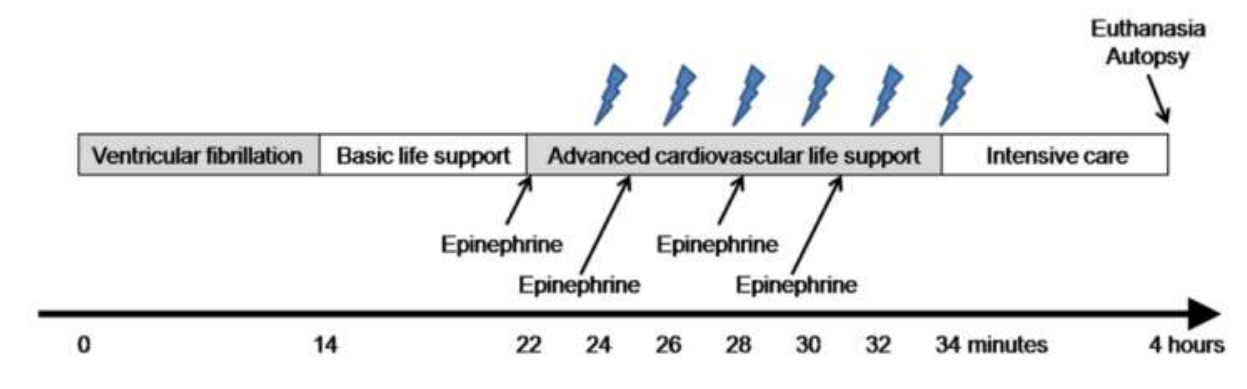

Fig. 2. Experimental timeline. Lighthing marks indicate the onset of a 10-second pause
in chest compressions for rhythm analysis and $150-$ s shock, if indicated.

Echocardiograms were obtained by an experienced investigator five minutes before induction of VF, immediately after initiation of $V F$, and in one-minute intervals during ACLS (Fig. 3). LV wall thickness and EDV during CPR were measured at the frame showing maximal chamber dimension of the LV following release of chest compression. EDV, which was the primary outcome measurement of the present study, was expressed as a percent of measured EDV relative to EDV at the onset of VF (\%EDV), to correct for its substantial inter-animal variation.

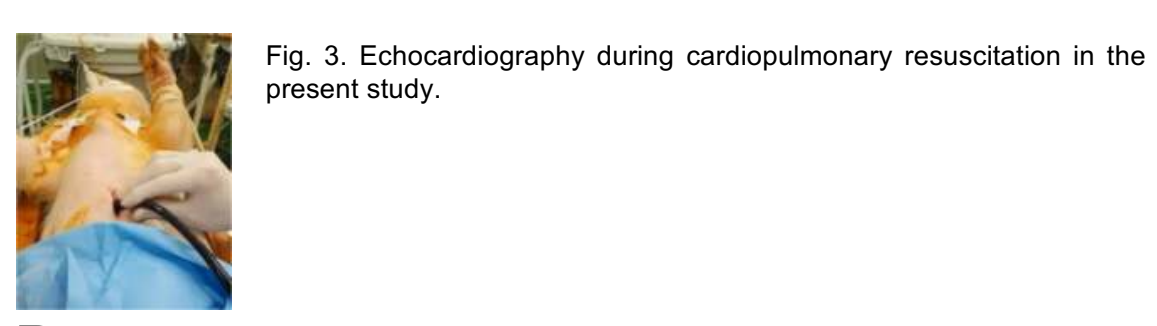

\section{Results}

Eight animals underwent the 12 minuteACLS period without achieving ROSC. Thus, a total of 96 sets of $\mathrm{ETCO}_{2}$, arterial pressure, LV wall thickness, and \%EDV data were collected (12 times with one-minute intervals in eight animals).
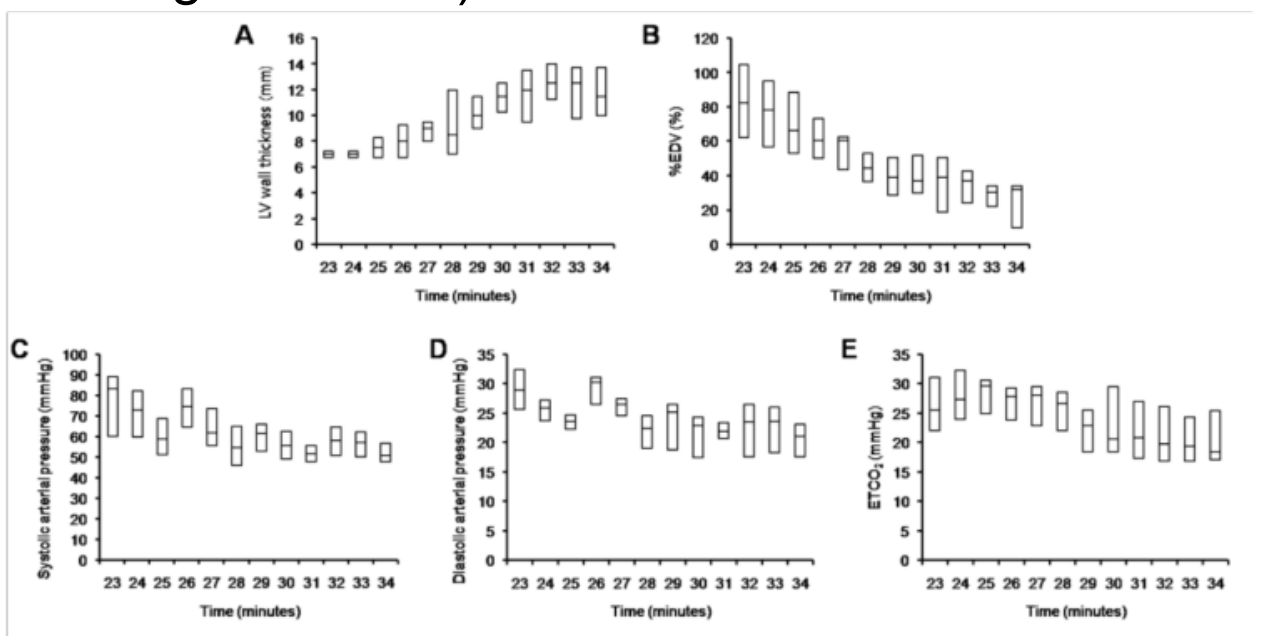

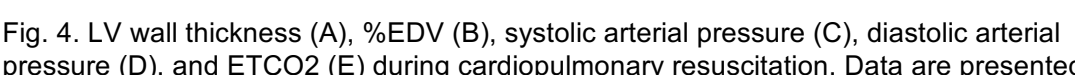

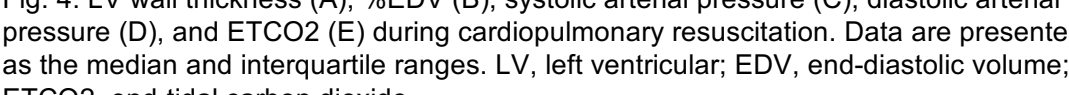

Each repeatedly measured response variable, including LV wall thickness and \%EDV, were significantly correlated with each predictor variable, including systolic and diastolic arterial pressure, and $\mathrm{ETCO}_{2}($ Table 1).

Table 1. Correlations between repeatedly measured variables

\begin{tabular}{|c|c|c|c|}
\hline Jariables & Correlation coefficient & $95 \% \mathrm{Cl}$ & $P$ \\
\hline \multicolumn{4}{|l|}{ LV wall thickness } \\
\hline $\mathrm{ETCO}_{2}$ & -0.55 & $-0.681--0.383$ & $<0.001$ \\
\hline jystolic arterial pressure & -0.476 & $-0.624--0.295$ & $<0.001$ \\
\hline Diastolic arterial pressure & -0.477 & $-0.625--0.297$ & $<0.001$ \\
\hline \multicolumn{4}{|l|}{$\begin{array}{l}\text { \%EDV } \\
\text { FDO }\end{array}$} \\
\hline $\mathrm{ETCO}_{2}$ & 0.303 & $0.098-0.483$ & 0.004 \\
\hline Systolic arterial pressure & 0.405 & $0.212-0.567$ & $<0.001$ \\
\hline Diastolic arterial pressure & 0.437 & $0.250-0.593$ & $<0.001$ \\
\hline
\end{tabular}

Tables 2 and 3 show the fixed effects of time and each predictor variable on LV wall thickness and \%EDV in linear mixed effect models, respectively. After adjustment for baseline covariates, systolic and diastolic arterial pressure were not predictive factors for LV wall thickness or \%EDV, while $\mathrm{ETCO}_{2}$

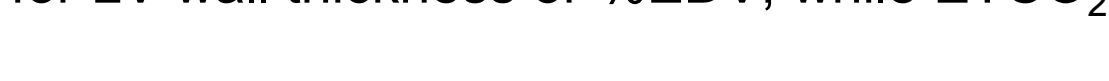

was a significant predictive factor for both LV wall thickness $(P=0.014)$ and $\% \operatorname{EDV}(P=$ 0.013).

Throughout our models, time was the most significant predictive factor for all response variables. For example, in the model including time and \%EDV, time explained $52.9 \%$ of total variance of \%EDV when adjusted for baseline covariates (semi-partial $R^{2}=0.529 ; 95 \%$ confidence interval $[\mathrm{Cl}]$, 0.407 - 0.642; $P<0.001$ ), while $\mathrm{ETCO}_{2}$ explained $14.2 \%$ of total variance of $\% E D V$ (semi-partial $\mathrm{R}^{2}=0.142 ; 95 \% \mathrm{Cl}=0.040-$ 0.283, $P=0.003$ ).

In conclusion, systolic and diastolic arterial pressure were not predictive factors for the severity of ischemic contracture. While $\mathrm{ETCO}_{2}$ was a significant predictive factor for the severity of ischemic contracture, it explained only a small proportion of the variance of ischemic contracture severity.

\begin{tabular}{|c|c|c|c|c|c|c|}
\hline & Coefficient & SE & $P$ & Semi-partial $R^{2}$ & $95 \% \mathrm{Cl}$ & \multirow{13}{*}{ 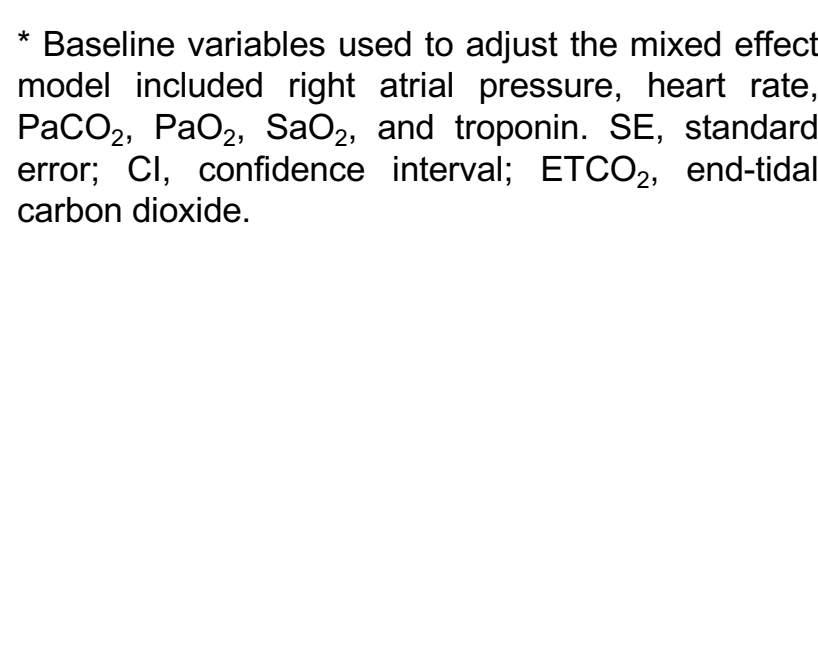 } \\
\hline Time & 0.514 & 0.044 & $<0.001$ & 0.405 & $0.269-0.538$ & \\
\hline $\mathrm{ETCO}_{2}(\mathrm{mmHg})$ & -0.069 & 0.043 & 0.11 & 0.056 & $0.002-0.173$ & \\
\hline Time* & 0.509 & 0.04 & $<0.001$ & 0.629 & $0.526-0.721$ & \\
\hline $\mathrm{ETCO}_{2}(\mathrm{mmHg})^{*}$ & -0.08 & 0.032 & 0.014 & 0.061 & $0.003-0.180$ & \\
\hline Time & 0.546 & 0.045 & $<0.001$ & 0.414 & $0.279-0.546$ & \\
\hline Systolic arterial pressure $(\mathrm{mmHg})$ & -0.004 & 0.014 & 0.782 & 0.001 & $0-0.056$ & \\
\hline Time* & 0.572 & 0.04 & $<0.001$ & 0.682 & $0.591-0.762$ & \\
\hline Systolic arterial pressure (mmHg)* & 0.011 & 0.009 & 0.225 & 0.015 & $0-0.100$ & \\
\hline Time & 0.525 & 0.045 & $<0.001$ & 0.403 & $0.268-0.537$ & \\
\hline Diastolic arterial pressure $(\mathrm{mmHg})$ & -0.049 & 0.042 & 0.248 & 0.012 & $0-0.091$ & \\
\hline Time* & 0.543 & 0.044 & $<0.001$ & 0.619 & $0.514-0.714$ & \\
\hline Diastolic arterial pressure $(\mathrm{mmHg})^{*}$ & -0.017 & 0.041 & 0.676 & 0.002 & $0-0.060$ & \\
\hline \multicolumn{6}{|c|}{ Table. 3. Fixed effects of time and hemodynamic parameters on \%EDV in mixed effect models } & \multirow{14}{*}{$\begin{array}{l}\text { * Baseline variables used to adjust the mixed effec } \\
\text { model included ETCO2 and troponin. SE, standar } \\
\text { error; Cl, confidence interval; ETCO2, end-tida } \\
\text { carbon dioxide. }\end{array}$} \\
\hline & Coefficient & $\mathrm{SE}$ & $P$ & Semi-partial $\mathrm{R}^{2}$ & $95 \% \mathrm{Cl}$ & \\
\hline Time & -5.512 & 0.402 & $<0.001$ & 0.410 & $0.275-0.543$ & \\
\hline $\mathrm{ETCO}_{2}(\mathrm{mmHg})$ & -1.239 & 0.399 & 0.003 & 0.146 & $0.043-0.288$ & \\
\hline Time* & -5.477 & 0.4 & $<0.001$ & 0.529 & $0.407-0.642$ & \\
\hline $\mathrm{ETCO}_{2}(\mathrm{mmHg})^{*}$ & -1.174 & 0.389 & 0.003 & 0.142 & $0.040-0.283$ & \\
\hline Time & -5.032 & 0.424 & $<0.001$ & 0.377 & $0.240-0.514$ & \\
\hline Systolic arterial pressure $(\mathrm{mmHg})$ & -0.114 & 0.129 & 0.378 & 0.008 & $0-0.082$ & \\
\hline Time* & -4.961 & 0.418 & $<0.001$ & 0.491 & $0.363-0.611$ & \\
\hline Systolic arterial pressure $(\mathrm{mmHg})^{*}$ & -0.074 & 0.123 & 0.549 & 0.006 & $0-0.074$ & \\
\hline Time & -4.719 & 0.421 & $<0.001$ & 0.345 & $0.209-0.486$ & \\
\hline Diastolic arterial pressure $(\mathrm{mmHg})$ & 0.198 & $\begin{array}{l}0.395 \\
0.395-10\end{array}$ & 0.617 & 0.002 & $0-0.060$ & \\
\hline Time* & -4.699 & 0.42 & $<0.001$ & 0.454 & $0.322-0.580$ & \\
\hline Diastolic arterial pressure $(\mathrm{mmHg})^{*}$ & 0.234 & 0.391 & 0.552 & 0.003 & $0-0.066$ & \\
\hline
\end{tabular}

